\title{
Statistical Properties of Residual Stresses and Intergranular Fracture in Ceramic Materials
}

M. Ortiz

Mem. ASME:

\section{S. Suresh}

Mem. ASME.

Division of Engineering, Brown University, Providence, RI 02912
The problem addressed in this paper concerns the statistical characterization of the state of residual stress generated in polycrystalline ceramics during cooling from the fabrication temperature. Detailed finite element simulations are carried out for an ensemble of large numbers of randomly oriented, planar hexagonal grains with elastic and thermal expansion anisotropy, and brittle grain interfaces. The calculations show that the distribution of normal and shear tractions induced by thermal contraction mismatch among grains is gaussian and that these tractions are statistically independent random variables. Although the gaussian nature of the distributions remains unaffected by the introduction of elastic anisotropy, the results indicate that elastic anisotropy has a significant effect on the residual stresses for finite departures from isotropy. When the hexagonal grains are randomly distorted, the magnitude and distribution of residual stresses are found to be insignificantly altered. Spontaneous microfracture due to the generation of internal stresses is also simulated in the analysis by allowing for the nucleation and growth of intergranular microcracks when the fracture energy along the grain facets exceeds a certain critical value. When such microcracking is incorporated into the computation, the levels of residual stress are markedly reduced as a consequence of stress dissipation. The dependence of intergranular microcracking on grain size and temperature variation is examined and the predicted trends on material degradation or the complete suppression of microfracture are discussed in the light of available experimental results.

\section{Introduction}

In noncubic, single-phase polycrystalline aggregates and in multiphase mixtures of ceramic materials, cooling from the processing temperature gives rise to microstructural residual stresses. These internal stresses, induced by the anisotropy of thermal contraction between adjacent grains, can promote spontaneous cracking along grain facets (e.g., Clarke, 1964; Matsuo and Sasaki, 1966; Davidge and Green, 1968; Kuszyk and Bradt, 1973; Cleveland and Bradt, 1978; Evans, 1978; Blendell and Coble, 1982; Yu and Evans, 1985; Fredrich and Wong, 1986). The occurrence of microcracking has been found to depend on the scale of the microstructure in that there exists a critical grain size in single-phase systems below which grain boundary microfracture is essentially suppressed (Matsuo and Sasaki, 1966; Evans, 1978; Rice and Pohanka, 1979). In addition, prior studies have identified the existence of a critical grain size beyond which total loss of material integrity occurs

Contributed by the Applied Mechanics Division of ThE AmERICAN SocietY of Mechanical. Engineers for publication in the ASME Journal of APPLIED NeCHANics.

Discussion on this paper should be addressed to the Technical Editor, ProIessor Lewis T. Wheeler, Department of Mechanical Engineering, University of Houston, Houston, TX 77204-4792, and will be accepted until four months after inal publication of the paper itself in the ASME Journal of APPLIED MEchivics.

Manuscript received by the ASME Applied Mechanics Division, May 14, 1991; final revision; Dec. 3, 1991. Associate Technical Editor: R. M. McMeeking. in some ceramic materials (Kuszyk and Bradt, 1973; Rice and Pohanka, 1979). The cooling rate is also known to have an effect on the magnitude of the microstructural stresses and on the extent of microcracking (Blendell and Coble, 1982).

On the basis of a study of residual stresses developed in polycrystalline metals during repeated heating and cooling, Boas and Honeycombe (1946) postulated that microstructural stresses are relatively insensitive to elastic anisotropy. In more recent analyses of intergranular residual stresses in thermally anisotropic, but elastically isotropic, aggregates of planar hexagonal grains, Evans (1978) and Fu and Evans (1985) developed closed-form solutions for the distribution of internal stresses along grain facets. It was shown that a singularity exists for stresses near a grain boundary triple point. By means of a perturbation expansion, Ortiz and Molinari (1988) showed that elastic anisotropy is indeed a second-order effect when deviations from elastic and thermal isotropy are small. They went on to determining analytically some of the statistical properties of the residual stress distribution such as their mean square value. Some caution must be exercised, however, in interpreting these results. It is known that perturbation expansions may not converge pointwise, particularly close to singularities. To ascertain the conditions which develop at triple point junctions, for instance, more detailed analyses of the type conducted by Tvergaard and Hutchinson (1988) are required. These 
investigators analyzed the stress singularities at triple points in planar arrays of hexagonal grains by an asymptotic method and by way of numerical simulation.

More recently, Laws and Lee (1989) have examined the evolution of residual stresses and microfracture in elastically isotropic and thermally anisotropic polycrystalline ceramics, the same case treated analytically by Ortiz and Molinari (1988). Laws and Lee (1989) have computed numerically the residual stress field for arbitrary orientation distributions of the axes of thermal anisotropy. They note that it is necessary to consider a two-dimensional polycrystalline ensemble of at least 200 grains in order to derive accurate predictions of the normal tractions at the midpoint of grain boundary facets.

Most of the analyses available to date on the generation of internal stresses by thermal and/or elastic anisotropy in brittle solids have been prediated upon specific choices of crystallographic orientations for neighboring grains within the polycrystalline ensemble (e.g., Evans, 1978; Fu and Evans, 1985; Tvergaard and Hutchinson, 1988). It is, however, evident that the random distribution of anisotropic grains in ceramic materials can give rise to residual stress patterns which may deviate markedly from those predicted by simple deterministic models. Furthermore, the limited number of grains for which analytical solutions of internal stresses and singularities have been discussed for some select cases of crystallographic anisotropy may not be representative of the trends exhibited by structural ceramics composed of large numbers of grains. Although analytical and numerical solutions of residual stress fields in polycrystalline aggregates consisting of large numbers of grains are beginning to emerge (e.g., Tvergaard and Hutchinson, 1988; Laws and Lee, 1989), the statistical properties of residual stress fields arising from thermal and elastic anisotropy remain largely unexplored.

In the present study, detailed finite element simulations have been carried out with the objective of investigating the statistical properties of the residual stress fields extending over large numbers of randomly oriented grains in single-phase brittle materials. The results provide valuable insights into the dependence of those properties on elastic and thermal anisotropy, grain size, and temperature. Furthermore, the nucleation and growth of grain boundary microcracks due to the development of residual stresses have also been analyzed, and the effects of grain size and thermal excursions on the extent of progressive microfracture have been investigated. In view of the present limitations of computational hardware, large-scale simulations must a fortiriori be based on two-dimensional models. It is expected, however, that many of the trends inferred from the two-dimensional analysis may carry over to the three-dimensional case as well.

\section{Problem Formulation and Numerical Modeling}

Throughout this study, we idealize a monolithic ceramic as an ensemble of randomly oriented, elastically and thermally anisotropic grains with brittle intergranular interfaces. We neglect any stress redistribution which may occur at high temperatures as a consequence of creep. Envision a process of cooling whereby the solid is brought from its fabrication temperature down to some final conditions, typically room temperature. The cooling is assumed to be slow enough that the temperature remains nearly uniform over the body at all times. During this cooling process, the body develops microstructural residual stresses due to the thermal and elastic anisotropy of grains. Our primary goal in this study is to develop an understanding of the statistical properties of the residual stress and microcrack distributions over large numbers of grains.

2.1 Modeling of Elastic and Thermal Properties. Under the assumptions of the analysis, the response of each grain is characterized by its elastic moduli $C_{i j k l}$ and its thermal expan-
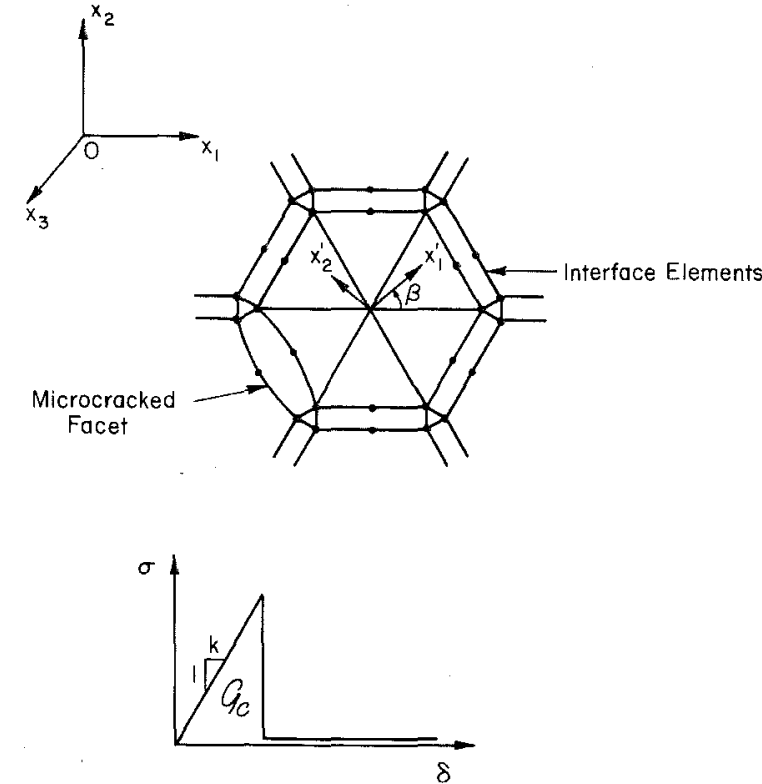

Fig. 1 Finite element model of hexagonal grain, showing connectivity with adjacent grains by means of interface elements. Inset is a schematic of the cohesive law obeyed by the interface elements. Also shown are the global and local grain cartesian axes. $x_{3}^{\prime}=x_{3}$.

sion coefficients $\alpha_{i j}$. The components of these tensors on some global cartesian reference frame $\left\{x_{1}, x_{2}, x_{3}\right\}$ depend on the orientation of the grain. The dependence on grain orientation can be characterized by introducing a set of axes $\left\{x_{1}^{\prime}, x_{2}^{\prime}, x_{3}^{\prime}\right\}$ attached to the crystalline lattice, Fig. 1. Then, if $C_{i j k l}^{\prime}$ and $\alpha_{i j}^{\prime}$ denote the crystal thermal moduli and thermal expansion coefficients, respectively, the global components are given by

$$
\begin{aligned}
& C_{i j k l}=Q_{i m} Q_{j n} Q_{k p} Q_{l q} C_{m n p q}^{\prime} \\
& \alpha_{i j}=Q_{i m} Q_{j n} \alpha_{m n}^{\prime}
\end{aligned}
$$

where $Q_{i j}$ is the appropriate transformation tensor. As indicated above, $Q_{i j}$ is taken as a uniformly distributed random variable. With these definitions, the stress-strain relations at the grain level may be expressed as

$$
\epsilon_{i j}^{\prime}=C_{i j k l}^{\prime} \sigma_{k l}^{\prime}+\theta \alpha_{i j}^{\prime}
$$

where $\epsilon_{i j}^{\prime}$ and $\sigma_{i j}^{\prime}$ denote the components of strain and stress relative to the grain axes, respectively, and $\theta$ is the drop in temperature.

We confine our attention to a consideration of the relative roles played by elastic and thermal anisotropy in the generation of residual stresses and microcracking. In particular, we seek to ascertain whether the effect of elastic anisotropy is significant for finite departures from isotropy. To this end, a simple model proposed by Tvergaard and Hutchinson (1988) is employed. In the present notation, this model amounts to taking $C_{i j k l}^{\prime}=\lambda \delta_{i j} \delta_{k l}+\mu\left(\sigma_{i l} \delta_{j k}+\delta_{i k} \delta_{j l}\right)$

$$
+(f-1)(\lambda+2 \mu) \delta_{1 i} \delta_{1 j} \delta_{1 k} \delta_{1 l}+\left(f^{-1}-1\right)(\lambda+2 \mu) \delta_{2 i} \delta_{2 j} \delta_{2 k} \delta_{2 l}
$$

where $\lambda$ and $\mu$ are Lamé-like constants and $f$ is an additional material parameter. For $f=1$, the moduli are isotropic. For $f \neq 1$, the crystals become elastically orthotropic, with $\left\{x_{1}\right.$, $\left.x_{2}^{\prime}, x_{3}^{\prime}\right\}$ as the principal axes of orthotropy. The quantity $\log (f)$ may conveniently be taken as a measure of anisotropy of the crystals.

The moduli (3) are designed for use in two-dimensional calculations, with $x_{3}=x_{3}^{\prime}$ as the out-of-plane direction. Tvergaard and Hutchinson (1988) noted that (3) does not model the elasticities of any particular ceramic, but that, choosing $f=\sqrt{2}$ gives a ratio of $C_{1111}^{\prime} / C_{2222}^{\prime}=2$, which reflects the typical properties of a number of crystals (Simmons and Wang, 1971). 
We note that the moduli (3) are orthotropic, with $x_{3}^{\prime}$ as one of the principal axes of orthotropy. Under these conditions, it is convenient to identify the grain axes with the principal axes of orthotropy. It is further assumed that the thermal expansion tensor $\alpha_{i j}^{\prime}$ has the same principal axes as the elastic orthotropy, and the principal thermal expansion coefficients are denoted by $\alpha_{1}, \alpha_{2}$, and $\alpha_{3}$.

In a two-dimensional setting, the orientations of the grains are defined by a single angle $\beta$ such that

$$
\mathbf{Q}=\left(\begin{array}{ccc}
\cos \beta & -\sin \beta & 0 \\
\sin \beta & \cos \beta & 0 \\
0 & 0 & 1
\end{array}\right)
$$

Throughout our simulations, we have taken $\beta$ to be a random variable uniformly distributed in the range $[0, \pi]$. In actual finite element computations we have defined the orientations of individual grains using a random number generator.

All calculations presented in this paper are for plane-strain deformation, for which $\epsilon_{33}^{\prime}=\epsilon_{33}=0$. This condition can be used, in conjunction with the orthotropy of the grains, to reduce (2) to the two-dimensional form

$$
\begin{aligned}
& \epsilon_{11}^{\prime}=\left(C_{11 \alpha \beta}^{\prime}-\frac{C_{1133}^{\prime} C_{33 \alpha \beta}^{\prime}}{C_{3333}^{\prime}}\right) \sigma_{\alpha \beta}^{\prime}+\left(\alpha_{1}-\frac{C_{1133}^{\prime}}{C_{3333}^{\prime}} \alpha_{3}\right) \theta \\
& \epsilon_{22}^{\prime}=\left(C_{22 \alpha \beta}^{\prime}-\frac{C_{2233}^{\prime} C_{33 \alpha \beta}^{\prime}}{C_{3333}^{\prime}}\right) \sigma_{\alpha \beta}^{\prime}+\left(\alpha_{2}-\frac{C_{2233}^{\prime}}{C_{3333}^{\prime}} \alpha_{3}\right) \theta \\
& 2 \epsilon_{12}^{\prime}=C_{1212}^{\prime} \sigma_{12}^{\prime} .
\end{aligned}
$$

Some useful insights into the parametric dependencies of the solution can be derived as follows. The parameters which define the model are Young's modulus $E$, Poisson's ratio $\nu$, the dimensionless elastic anisotropy constant $f$, the temperature drop $\theta$, the tensile strength of the facets $\sigma_{c}$, and the principal thermal expansion coefficients $\alpha_{1}, \alpha_{2}$, and $\alpha_{3}$. However, these latter parameters and the temperature enter the formulation only through the combinations involved in (5).

Introduce the reduced coefficients

$$
\alpha_{1}^{*}=\alpha_{1}-\frac{C_{1133}^{\prime}}{C_{3333}^{\prime}} \alpha_{3}, \alpha_{2}^{*}=\alpha_{2}-\frac{C_{2233}^{\prime}}{C_{3333}^{\prime}} \alpha_{3} .
$$

By the assumed isotropy of the distribution of grain orientations, it follows that $\alpha_{1}^{*}, \alpha_{2}^{*}$, and $\theta$ can only enter the solution through the combination $\left|\alpha_{1}^{*}-\alpha_{2}^{*}\right| \theta \equiv \alpha_{e} \theta$, where the effective thermal expansion coefficient $\alpha_{e}$ may be taken as a measure of the degree of thermal anisotropy of the grains. For moduli (3), it follows that $C_{1133}^{\prime}=C_{2233}^{\prime}=\lambda$, and the effective thermal expansion coefficient reduces to

$$
\alpha_{e}=\left|\alpha_{1}-\alpha_{2}\right| \text {. }
$$

We note that $\alpha_{e}=0$ if the grains are thermally isotropic.

2.2 Computational Model. In order to be able to make statistical inferences regarding the distribution of residual stresses, a large number of grains needs to be included in the analysis. This effectively rules out the possibility of conducting three-dimensional numerical simulations. Even in two dimensions, an idealized description of each individual grain needs to be adopted. In our calculations the polycrystalline ensemble is assumed to be topologically hexagonal (Fig. 1). Each grain is discretized into six four-noded elements, with quadratic displacement interpolation over the grain boundary. In some simulations, we have randomly distorted the grains with a view to studying the sensitivity of the results to grain geometry. This distortion is accomplished by displacing the nodes along random directions by a prescribed distance, typically some fraction of the facet length.

Adjacent grains are held together by means of three-noded surface elements (Fig. 1). These elements allow for quadratic opening displacements. The grain boundaries are assumed to be brittle and to crack according to the cohesive law shown in Fig. 1. This is linear in both the normal and shearing tractions up to a critical value of the former, at which point the strength of the boundary is assumed to drop to zero. The shaded area under the tensile cohesive law in Fig. 1 defines the critical energy release rate $G_{c}$ in the absence of any shearing. The stiffness $k$ of the interface is chosen to be the same in tension and in shear, and to be much larger than that of the surrounding grains. Under these conditions, the grain boundaries remain essentially undeformed up to decohesion. The cohesive law is enforced on three quadrature points of each surface element. At any given point in the solution, an element can simultaneously contain failed and intact quadrature points. This permits the simulation of partially fractured boundary facets. We also note that microcracks need not remain confined to a facet, but can extend across triple grain junctions into adjacent facets.

The calculations proceed incrementally. At each increment, the temperature is decreased by a prescribed amount. An iteration is then carried out until equilibrium is satisfied and the microcracking process has stabilized.

2.3 Sample Size Requirements. A key consideration in designing the numerical model concerns the number of grains required to accurately predict the various statistics of interest. Imagine that stresses are sampled at $n$ points within the region of the solid under consideration. In calculations, the sampling points coincide with the quadrature points of the element. Denote by $\sigma_{\alpha \beta}^{i}$ the components of stress at sampling point $i$. By virtue of the randomness in the orientations of the grains, the pointwise values of the components of stress are themselves random variables of an as yet unknown distribution. By equilibrium, the average stresses

$$
\left\langle\sigma_{\alpha \beta}\right\rangle=\frac{1}{n} \sum_{i=1}^{n} \sigma_{\alpha \beta}^{i}
$$

must vanish identically. Let

$$
\left\langle\sigma_{\alpha \beta} \sigma_{\gamma \delta}\right\rangle=\frac{1}{n} \sum_{i=1}^{n} \sigma_{\alpha \beta}^{i} \sigma_{\gamma \delta}^{i}
$$

be the covariance matrix of the (pointwise) stresses. Analytical expressions for $\left\langle\sigma_{\alpha \beta} \sigma_{\gamma \delta}\right\rangle$ were obtained by Ortiz and Molinari (1988) for the case of thermally anisotropic but elastically isotropic solids. By the isotropy of the stress distribution it follows that $\left\langle\sigma_{\alpha \beta} \sigma_{\gamma \delta}\right\rangle$ must be an isotropic tensor, i.e., must be of the form

$$
\left\langle\sigma_{\alpha \beta} \sigma_{\gamma \delta}\right\rangle=\left(\sigma^{2}-2 \tau^{2}\right) \delta_{\alpha \beta} \delta_{\gamma \delta}+\tau^{2}\left(\delta_{\alpha \delta} \delta_{\beta \gamma}+\delta_{\alpha \gamma} \delta_{\beta \delta}\right)
$$

where

$$
\begin{aligned}
& \sigma=\left\langle\sigma_{11} \sigma_{11}\right\rangle^{1 / 2}=\left\langle\sigma_{22} \sigma_{22}\right\rangle^{1 / 2} \\
& \tau=\left\langle\sigma_{12} \sigma_{12}\right\rangle^{1 / 2}
\end{aligned}
$$

are the standard deviations of the normal and shear tractions, respectively. These quantities provide scalar measures of the magnitude of the residual stresses. A further consequence of the isotropy of the covariance matrix is that $\left\langle\sigma_{11} \sigma_{12}\right\rangle$ $=\left\langle\sigma_{22} \sigma_{12}\right\rangle=0$, i.e., the normal and shear stress components are uncorrelated pointwise.

The values of $\sigma$ and $\tau$ corresponding to different microstructural realizations also define random variables. In actual simulations, however, only one particular grain arrangement, i.e., one single sample from the overall ensemble of polycrystalline bodies, is analyzed, and $\sigma$ and $\tau$ are computed as in (9). A central question in sampling theory is how representative the parametric estimates so computed are of their respective ensemble averages. In general, the distributions of $\sigma$ and $\tau$ may be expected to become increasingly more concentrated about their means as the size $n$ of the sample (i.e., the number of grains included in the simulation) is increased. Thus, the larger 
the choice of $n$, the more likely the computed values of $\sigma$ and $\tau$ are to be close to their mean values.

Next, we wish to determine how large $n$ needs to be for the mean and standard deviation of $\sigma$ and $\tau$ to be within acceptable confidence intervals. Simple estimates of sample size requirements may be obtained from standard results of parametric point estimation (see, for example, Mood et al., 1974). Consider a random sample $\left(X_{1}, \ldots, X_{n}\right)$ of size $n$ of a normally distributed variable $X$ with mean $m_{1}$ and standard deviation $m_{2}$. Then, the maximum likelihood estimators $M_{1}$ and $M_{2}$ of $m_{1}$ and $m_{2}$ are given by the familiar expressions

$$
M_{1}=\frac{1}{n} \sum_{i=1}^{n} X_{i}, \quad M_{2}=\frac{1}{n} \sum_{i=1}^{n}\left(X_{i}-M_{1}\right)^{2},
$$

respectively. Evidently, different finite samples may be expected to yield different values of $M_{1}$ and $M_{2}$. In fact, the values of $M_{1}$ and $M_{2}$ so obtained are themselves random variables. A particularly useful result is that, as the size of the sample $n \rightarrow \infty, M_{1}$ and $M_{2}$ are asymptotically normally distributed with mean $m_{1}$ and $m_{2}$, respectively, and variance

$$
s_{1}^{2}=m_{2} / n, s_{2}^{2}=2 m_{2}^{2} / n,
$$

respectively. These standard deviations coincide with the socalled Cramér-Rao lower bounds (Mood et al., 1974).

Next, we apply these results to the residual stress distribution. As it will become apparent in subsequent discussions, $\sigma_{\alpha \beta}$ turn out to be very nearly normally distributed and uncorrelated. Hence, it follows from the above results that the maximum likelihood estimators (9) of $\sigma$ and $\tau$ are themselves normally distributed with mean

$$
m_{\sigma}=\sigma, m_{\tau}=\tau
$$

and standard deviation

$$
s_{\sigma}^{2}=2 \sigma^{2} / n, s_{\tau}^{2}=2 \tau^{2} / n .
$$

From tables of the normal distribution, it is found that the computed values of $\sigma$ and $\tau$ may be expected to lie in the intervals

$$
\begin{aligned}
& m_{\sigma}-1.96 s_{\sigma} \leq \sigma \leq m_{\sigma}+1.96 s_{\sigma} \\
& m_{\tau}-1.96 s_{\tau} \leq \tau \leq m_{\tau}+1.96 s_{\tau},
\end{aligned}
$$

with 95 percent confidence. The implication of (16) is that, if we wish to obtain computed values of $\sigma$ and $\tau$ within, say, $p$ percent of the averages $m_{\sigma}$ and $m_{\tau}$ with 95 percent confidence, then we must have

$$
s_{\sigma} \leq 0.51 \mathrm{pm}_{\sigma} \quad s_{\tau} \leq 0.51 \mathrm{pm}_{\tau} .
$$

Using (14) and (15), we obtain the condition

$$
n \geq 2 /(0.51 p)^{2}
$$

If, for instance, we wish to impose a tolerance of ten percent on the results, i.e., $p=0.1$, then a model containing $n \geq 769$ facets is required. This corresponds to an ensemble of 331 grains, or 4,303 nodal points. If the tolerance is lowered to one percent, then the number of facets must be increased to $n=76,894$-clearly out of the realm of possibility for the present investigation. Throughout our calculations we have used a model containing 469 grains and 1,332 facets. The estimated standard deviations so obtained are within 7.6 percent of their mean values with 95 percent confidence. The finite element mesh comprises 4,146 solid elements and 6,097 nodal points.

\section{Results of Numerical Simulations}

3.1 Effect of Thermal Anisotropy. Our first simulations are aimed at characterizing the state of residual stress that develops in the linear range, i.e., in the absence of microcracking. By virtue of the linearity of the problem, the residual stresses are then proportional to the temperature drop. We start by considering the case of thermally anisotropic but elastically isotropic grains. This corresponds to taking $f=1$ in (3) The properties of the material used in the calculations are: $E=380 \mathrm{GPa}, \nu=0.25, \alpha_{1}=8.3 \times 10^{-6}{ }^{\circ} \mathrm{C}^{-1}, \alpha_{2}=9.0 \times 10^{-6}$ ${ }^{\circ} \mathrm{C}^{-1}$, grain size $d=25 \mu \mathrm{m}$, and temperature drop $\theta=1000^{\circ} \mathrm{C}$. For these parameters one has $\alpha_{e}=0.7 \times 10^{-6}{ }^{\circ} \mathrm{C}^{-1}$ and $\mu=152$ $\mathrm{GPa}$. These parameters are representative of polycrystalline alumina (e.g., Kingery et al., 1976).

Figures 2(a-c) show the histograms of the stress components $\sigma_{11}, \sigma_{22}, \sigma_{12}$ computed at the quadrature points within the elements, as well as gaussian fits obtained through the standard formulas (12). As may be seen, the distributions are strikingly gaussian. The mean stresses are computed to be zero, as befits the self-equilibrated nature of the residual stress field. As expected from the isotropy of the distribution of grain orien tations, the correlation between normal and shear stress components is virtually zero. In addition, $\sigma_{11}$ and $\sigma_{22}$ must be distributed identically. Under these conditions, the pointwise distribution of the residual stresses is fully described by the parameters $\sigma$ and $\tau$ defined above. These parameters may be thought of as mean-square values of the normal and shear stresses, respectively, and thus arise as natural residual stress measures.

As a test of the adequacy of the mesh size and the resulting traction samples obtained in the simulations, we have repeated the above calculations for a larger mesh of 4326 solid elements and 9373 nodal points. This corresponds to an ensemble of 721 grains containing 2070 facets. The standard deviations computed from the coarser mesh were within five percent of those computed from the finer mesh, which represents a statistically insignificant deviation from the results of the smaller model.

3.2 Effect of Thermal and Elastic Anisotropy. Next, we investigate the effect of elastic anisotropy by setting $f=1.414$ in the expression (3) for the elastic moduli. This choice of $f$ has been used by Tvergaard and Hutchinson (1988) in their study of stress singularities at triple point junctions, and defines a rather substantial departure from elastic isotropy not unlike those found in some ceramic single crystals (Simmons and Wang, 1971). The resulting residual stress histograms are shown in Fig. 3. It is evident from these results that the gaussian nature of the distributions remains unaffected by the intro duction of elastic anisotropy. As in the previous simulation, normal and shear stresses are found to be uncorrelated. However, the computed mean-square stresses are roughly three times larger than those computed under the assumption of elastic isotropy. These results indicate that, whereas thermal anisotropy may indeed be the main effect for small departures from thermal and elastic isotropy, as shown by Ortiz and Molinari (1988) for many actual polycrytalline materials the effect of elastic anisotropy cannot be neglected. All calculations presented in the sequel are for thermally as well as elastically anisotropic grains.

We also note the computed residual stresses are somewhat higher than those measured experimentally (e.g., Blendell and Coble, 1982, see discussions to follow). The assumption of no microcracking in the calculations is a contributing factor to this overshoot in the results. When microcracking is taken into account, the levels of stress are considerably reduced, as demonstrated in subsequent simulations. This underscores the important role played by microcracking as a stress dissipation mechanism.

3.3 Effect of Grain Distortion. The above calculations were carried out on an undistorted mesh, i.e., one in which all the grains are taken to be perfectly hexagonal. The results of the computations, however, are found to be quite insensitive to the degree of distortion of the mesh. If, for instance, the 


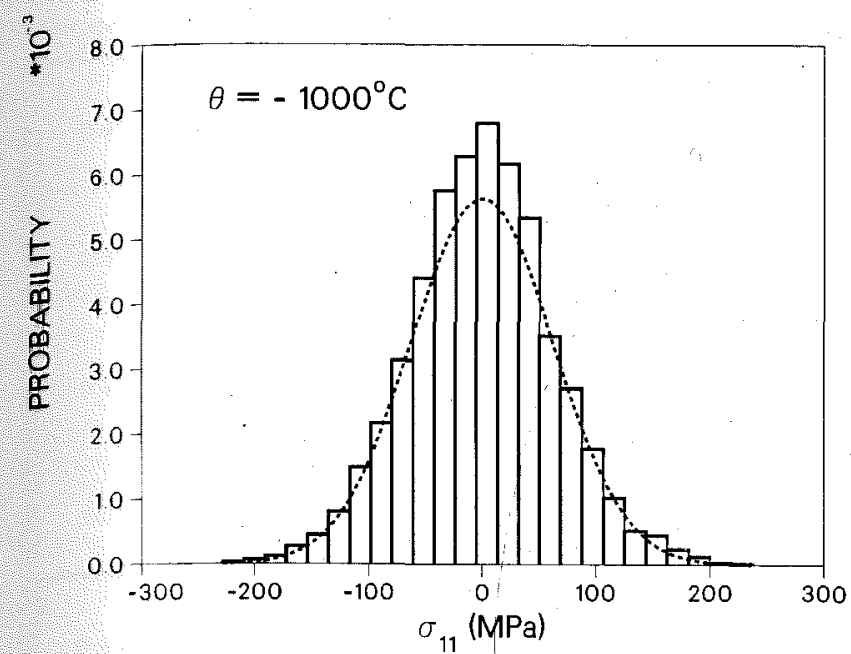

Fig. 2(a)

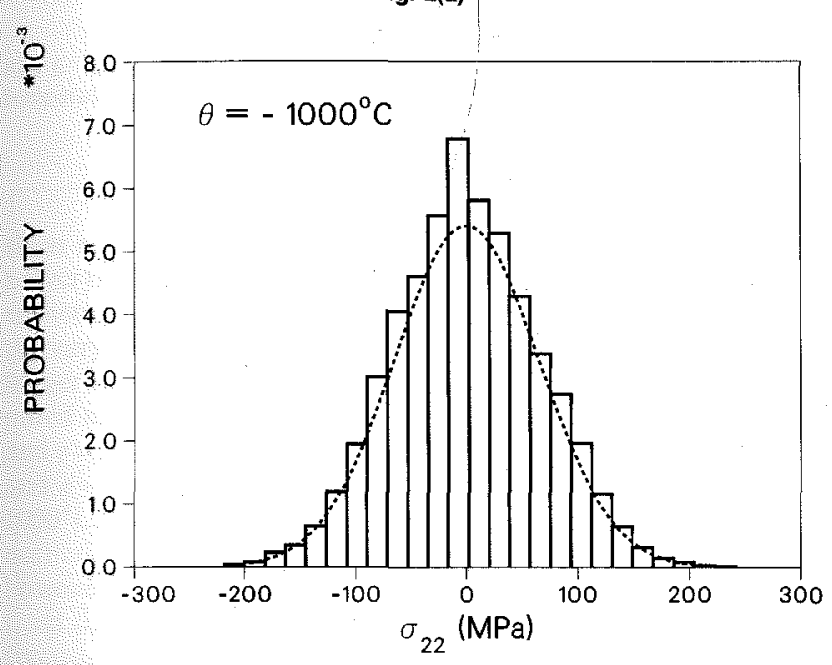

Fig. 2(b)

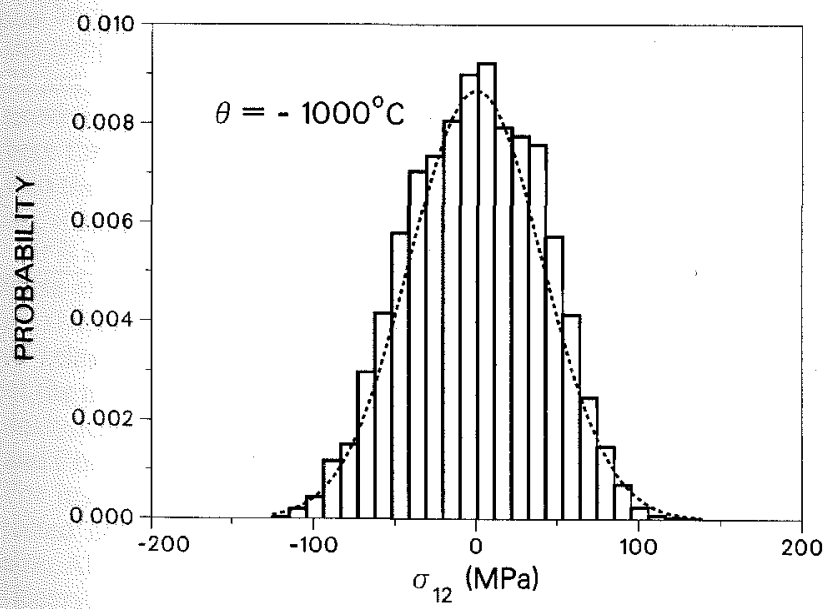

Fig. 2(c)

Fig. 2 Distribution of residual stresses induced by thermal anisotropy in the absence of elastic anisotropy and microcracking. Regular hexagonal mesh. Dashed lines represent gaussian fits to the computed distributions.

nodes defining the grain boundaries are displaced in random directions by one fifth of the facet length, the computed meansquare stresses are within six percent of those corresponding to the undistorted mesh. The histograms of normal and shear stress are also found to be ostensibly similar to those for the regular geometry. All remaining calculations are based on the perfectly hexagonal model.

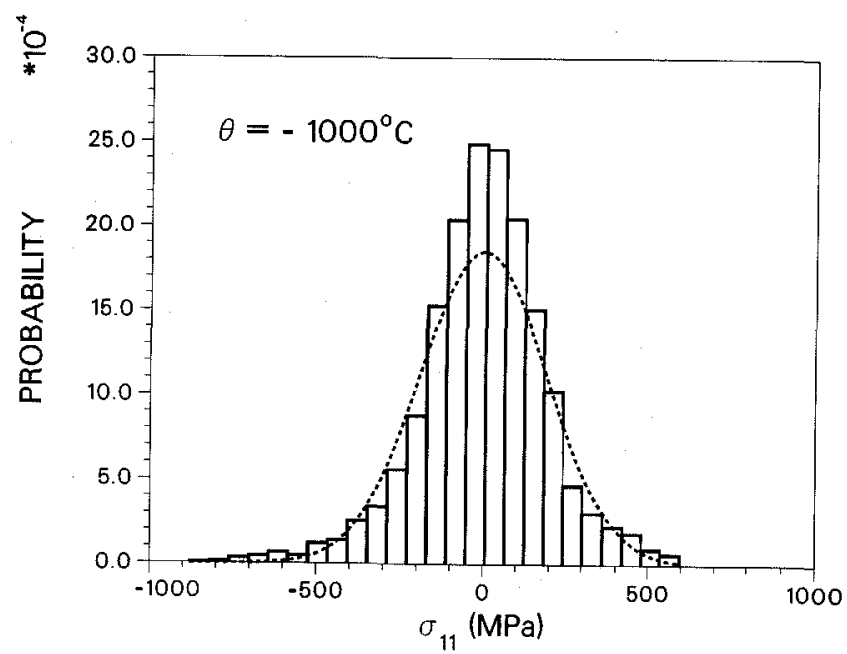

Fig. 3(a)

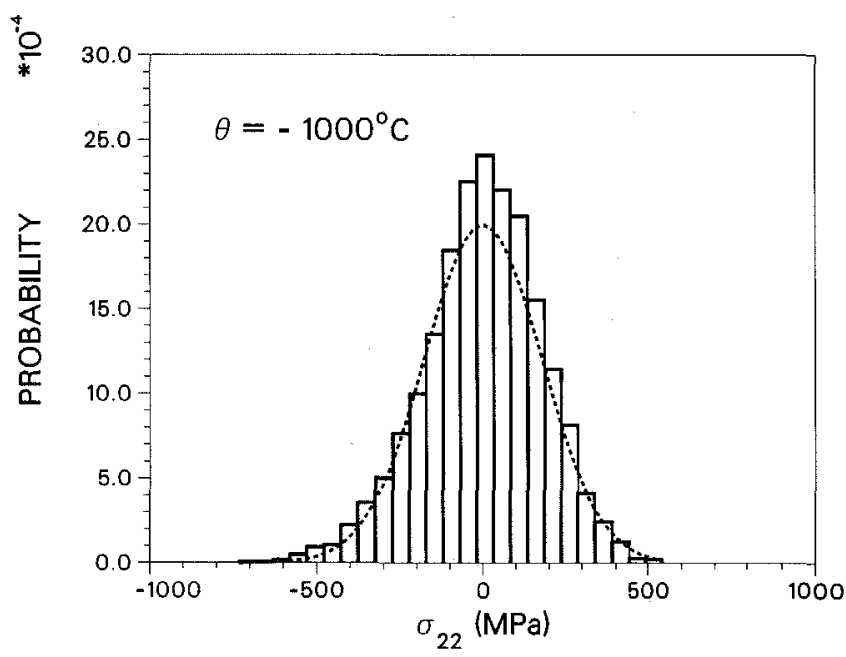

Fig. 3(b)

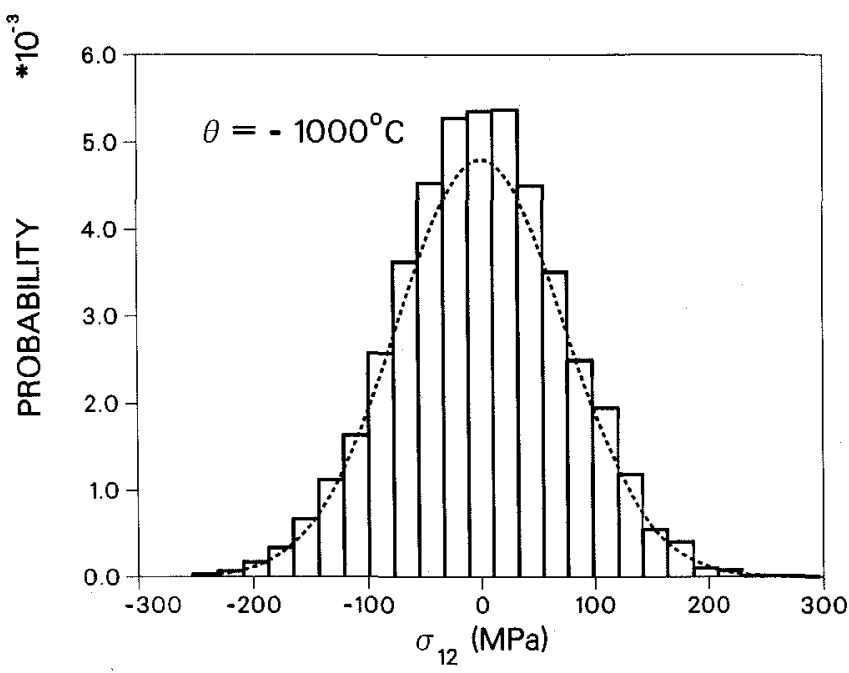

Fig. $3(c)$

Fig. 3 Distribution of residual stresses induced by thermal and elastic anisotropy in the absence of microcracking. Regular hexagonal mesh. Dashed lines represent gaussian fits to the computed distributions.

3.4 Grain Boundary Microcracking. Next, we extend the analysis into the nonlinear range by allowing for the formation of microcracks along grain boundaries. The surface energy $\gamma$ of the facets which, as discussed in Section 2.2, governs microcrack growth, is taken to be $1 \mathrm{~J} / \mathrm{m}$, which is typical of monolithic ceramics. Figure 4 shows the histograms of normal and 


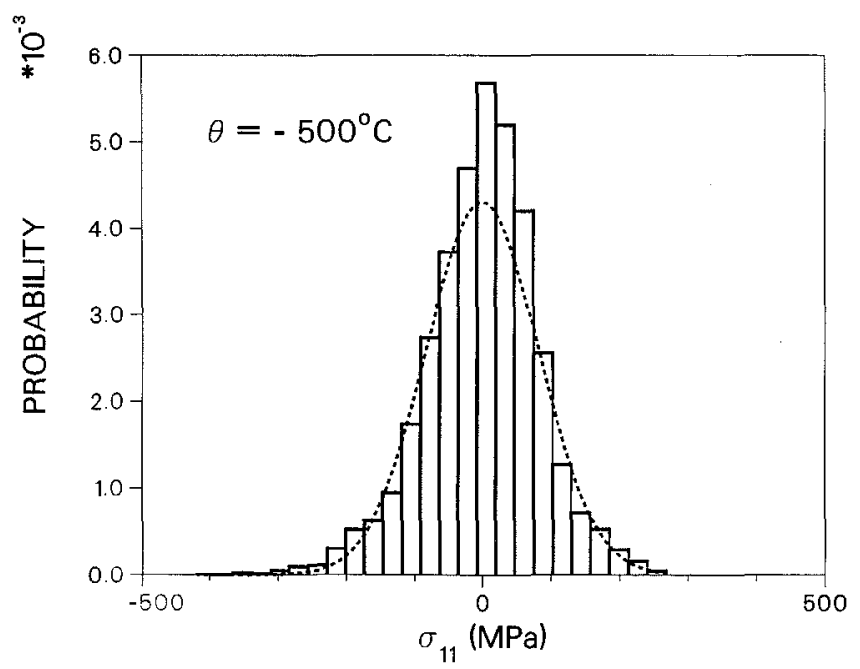

Fig. 4(a.1)

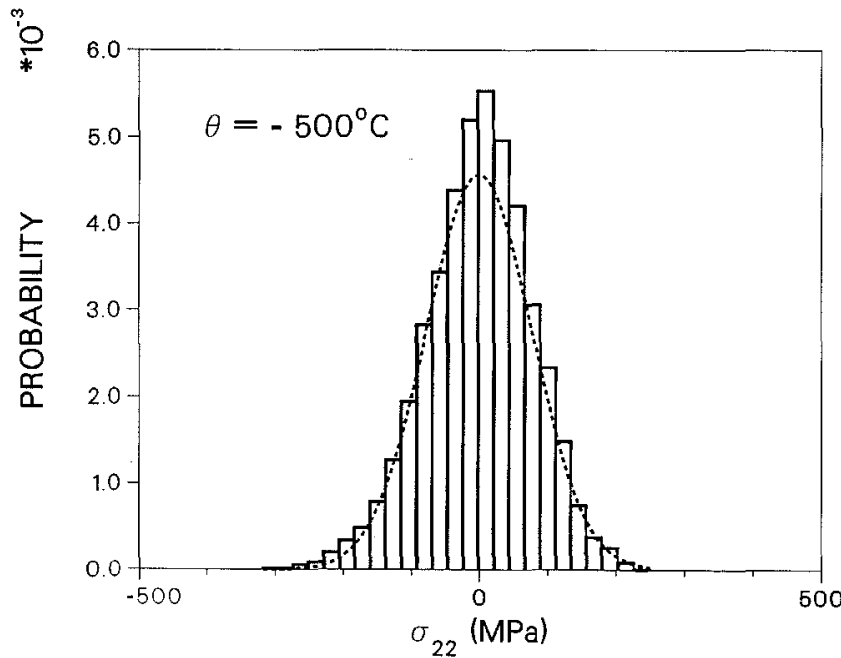

Fig. $4(a .2)$

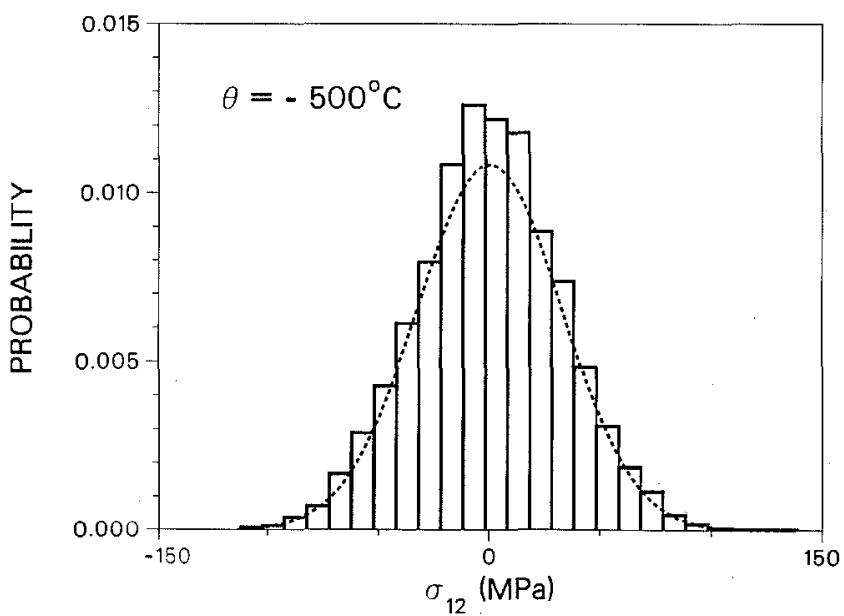

Fig. 4(a.3)

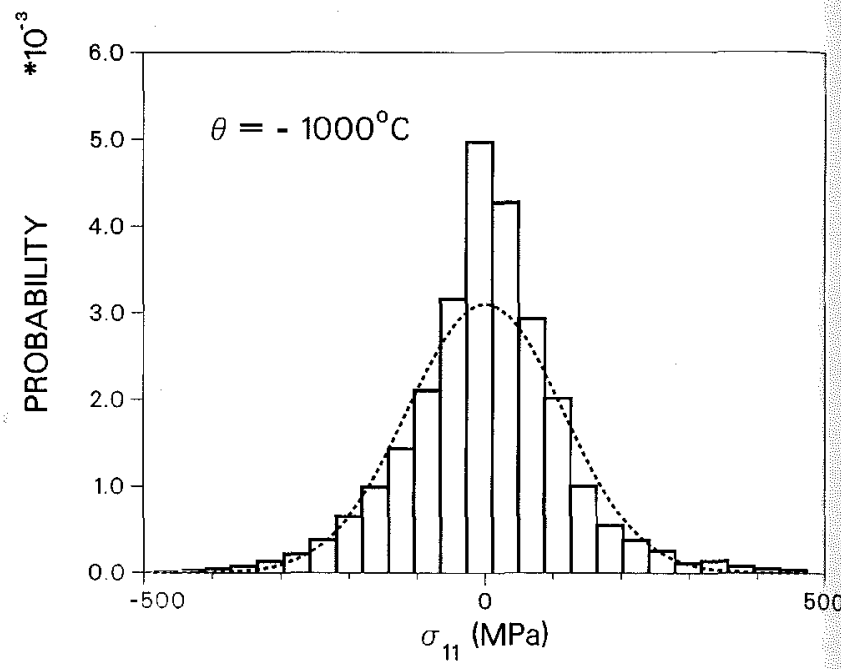

Fig. 4(b.1)

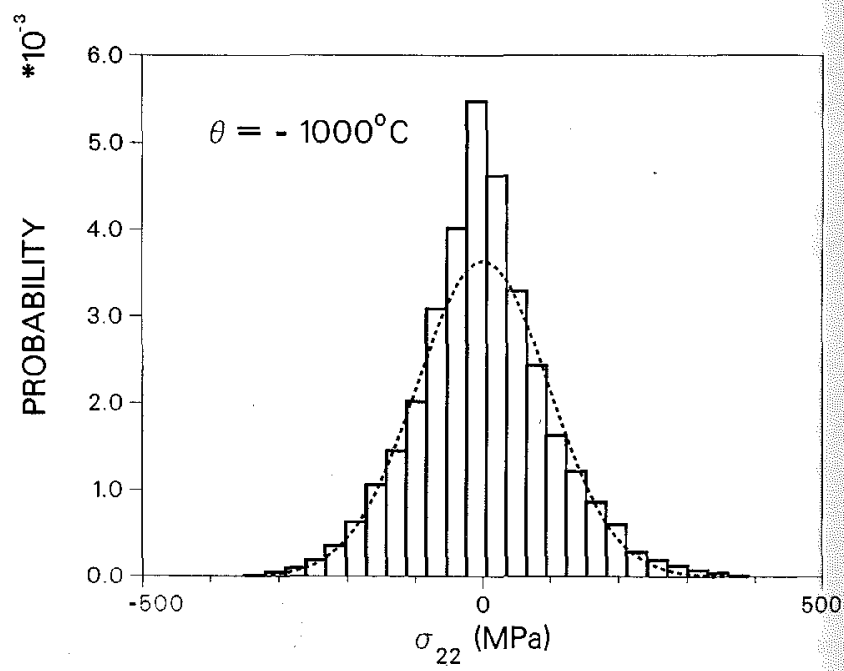

Fig. $\mathbf{4}(b .2)$

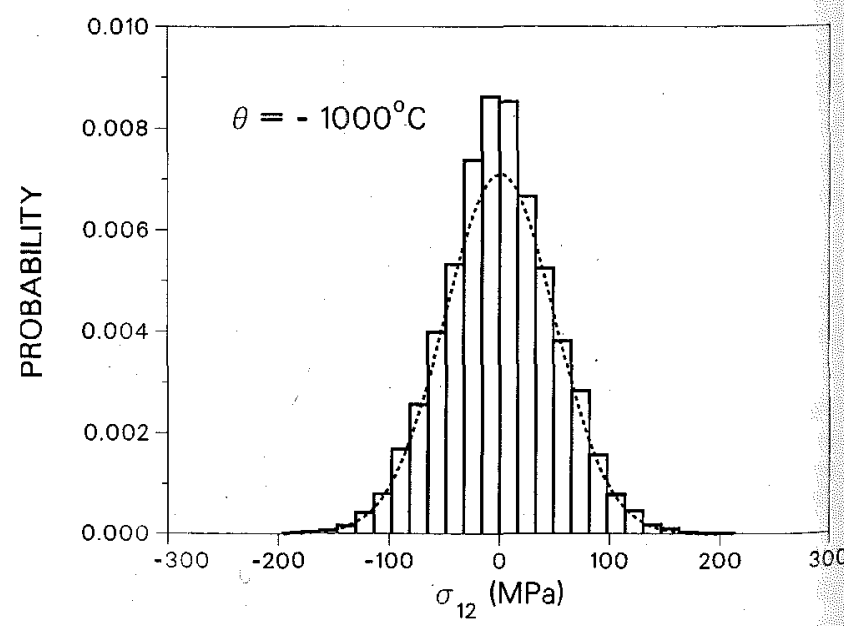

Fig. $4($ b.3)

Fig. 4 Distribution of residual stresses induced by thermal anisotropy, elastic anisotropy, and microcracking for two different temperature drops. Regular hexagonal mesh. Dashed lines represent gaussian fits to the computed distributions. 
MICROCRACK DENSITIES

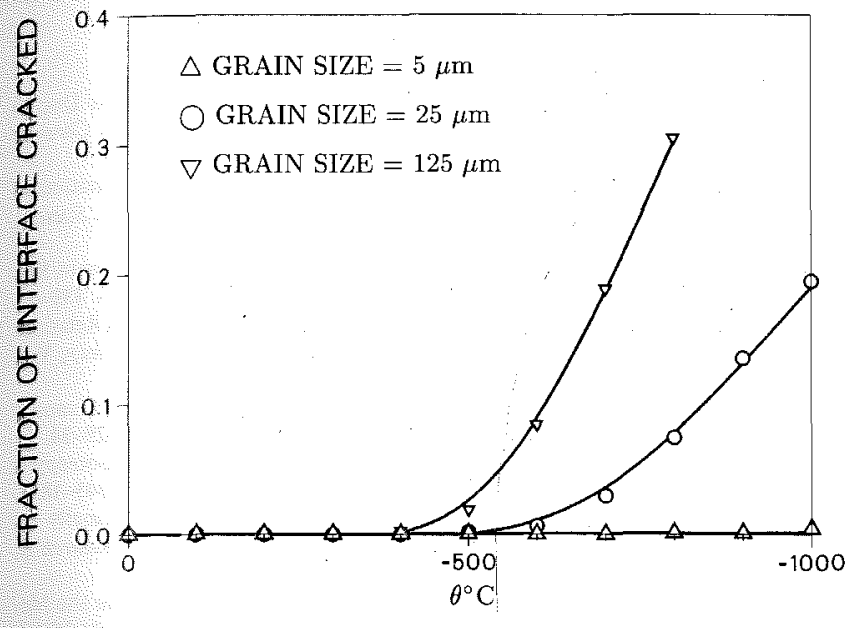

Fig. 5 Dependence of the fraction of cracked intergranular facets on temperature drop for three grain sizes

shear residual stress in a thermally and elastically anisotropic polycrystalline material $(f=1.414)$ subjected to temperature reductions of $500^{\circ} \mathrm{C}$ and $1000^{\circ} \mathrm{C}$. It is noted that, because of the varying extent of microcracking, the computed stresses no longer vary linearly with the temperature drop. Thus, the computed mean-square normal and shear stresses are $\sigma=91 \mathrm{MPa}$ and $\tau=37 \mathrm{MPa}$, respectively, for a temperature drop of $500^{\circ} \mathrm{C}$ and $o=121 \mathrm{MPa}$ and $\tau=56 \mathrm{MPa}$ for a temperature drop of $1000^{\circ} \mathrm{C}$. As before, the normal and shear stresses are ostensibly normally distributed and nearly uncorrelated. As expected, however, the mean-square residual stresses are sharply reduced from the values predicted for the case where no intergranular fracture is permitted. This result illustrates the important role played by microcracking in reducing the levels of residual stress.

The variation of the fraction of microcracked facets with the temperature drop is plotted in Fig. 5 for grain sizes $5 \mu \mathrm{m}$, $25 \mu \mathrm{m}$, and $125 \mu \mathrm{m}$. Contrary to the simulations discussed in previous sections which are insensitive to grain size effects, here the surface energy of the facets introduces a length scale into the problem, with the consequence that, after microcracking sets in, the results are markedly dependent on grain size.

It is apparent from Fig. 5 that, for a fixed temperature drop, there exists a critical grain size (in the present case, at least 5 $\mu \mathrm{m})$ below which grain boundary microcracking due to thermal and elastic anisotropy is completely suppressed. This prediction of the model is fully consistent with previous experimental and analytical results (Kuszyk and Bradt, 1973; Evans, 1978; Rice and Pohanka, 1979; Fu and Evans, 1985; Laws and Lee, 1989). For instance, Kuszyk and Bradt (1973) and Evans (1978) detected no appreciable microcracking below a threshold grain size. Also in keeping with the available observational evidence (Kuszyk and Bradt, 1973; Evans, 1978), for the grain size range $15-150 \mu \mathrm{m}$ the computed extent of microcracking increases rapidly with grain size. Indeed, Rice and Pohanka (1979) have observed considerable microcracking in alumina at grain sizes of about $100 \mu \mathrm{m}$. The behavior of very coarse grained systems varies from material to material. For some systems, beyond a certain grain size the rate at which microcracking increases with grain size slows down, probably due to saturation. Other systems spontaneously disintegrate as a result of extensive microfracture (Rice and Pohanka, 1979).

For the coarsest grained material considered in the calculations (grain size $=125 \mu \mathrm{m}$ ), some grains become completely detached following a temperature drop of the order of $800^{\circ} \mathrm{C}$, and the calculations have to be stopped. Thus, under the conditions of the analysis, spontaneous disintegration seems to occur prior to microcrack saturation. Whether or not disintegration precedes saturation in the numerical simulations is likely to depend critically on how triple grain joints are modeled and, more specifically, on the precise manner in which microcracks are allowed to propagate through triple points. For instance, if triple points are assumed to act as strong barriers to microcrack growth, then microcracks will tend to remain stably confined within isolated grain facets, and saturation will eventually be attained. Additionally, the two-dimensional nature of the model can be expected to facilitate the full detachment of grains, thus promoting disintegration.

\section{Discussion}

The finite element simulations conducted in this work provide detailed predictions of the magnitude and statistical distribution of thermal residual stresses in brittle solids. The implications of these results are now examined in the context of available experimental results for polycrystalline aggregates of noncubic ceramic materials.

For polycrystalline aluminum oxide with a grain size range of 50 to $150 \mu \mathrm{m}$, Blendell and Coble (1982) measured the internal stresses in the material at $77 \mathrm{~K}$ by means of the broadening of spectroscopic $\mathrm{R}$ lines following cooling from a temperature of $2150 \mathrm{~K}$ at constant cooling rates of 0.1 to $100 \mathrm{~K}$ / min. It has been suggested that there exists a freezing temperature below which the internal stresses build up during cooling without stress relaxation; this freezing temperature ( $\approx 1500 \mathrm{~K}$ for alumina) has been postulated to be independent of grain size and cooling rate (Blendell and Coble, 1982). Incorporating thermal and elastic anisotropy and allowing for spontaneous microcracking (and the attendant stress relaxation), the mean-square normal and shear residual stresses computed in the present study are $\sigma \approx 120 \mathrm{MPa}$ and $\tau \approx 55 \mathrm{MPa}$, respectively, for a grain size of $25 \mu \mathrm{m}$ and a temperature drop of $1000^{\circ} \mathrm{C}$. Given that the extent of microcracking increases rapidly with grain size, the level of residual stress at the grain size of $50 \mu \mathrm{m}$ considered by Blendell and Coble (1982) should be somewhat reduced from the level corresponding to a grain size of $25 \mu \mathrm{m}$ considered in our calculations. Indeed, the maximum value of residual stresses observed by Blendell and Coble (1982) was in the range 80 to $100 \mathrm{MPa}$ for the conditions investigated. Because measurements of microstructural residual stresses using spectroscopic methods are inherently prone to considerable experimental scatter and uncertainties, the agreement between the computed and measured residual stress levels may be considered satisfactory.

Our estimates of the dependence of microcrack density on the grain size also provide an opportunity for comparisons with experiment. The calculations show that, for alumina with a grain size smaller than $5 \mu \mathrm{m}$, essentially no microcracking occurs for a temperature drop of $1000^{\circ} \mathrm{C}$. It may then be inferred that thermally induced microfracture is essentially suppressed for fine-grained ceramics. The results of several independent experimental investigations on the threshold for spontaneous microcracking in ceramics are seen to be consistent with the trends predicted by the finite element calculations: (i) Evans (1978) examined the conditions for grain boundary microfracture due to thermal stresses in sintered (lucalox) $\mathrm{Al}_{2} \mathrm{O}_{3}$ that was annealed at various times to yield a range of grain sizes. For a temperature drop of $1850^{\circ} \mathrm{C}$, a threshold for the suppression of microcracking was identified. In transmission electron microscopy observations, no microcracks could be detected below an average grain size of about $80 \mu \mathrm{m}$. (ii) Kuszyk and Bradt (1973) and Cleveland and Bradt (1978) investigated the relationship between microcracking and grain size in three pseudobrookite oxides, $\mathrm{MgTi}_{2} \mathrm{O}_{5}, \mathrm{Fe}_{2} \mathrm{TiO}_{5}$, 
and $\mathrm{Al}_{2} \mathrm{TiO}_{5}$, which have a significantly greater degree of thermal anisotropy than polycrystalline $\mathrm{Al}_{2} \mathrm{O}_{3}$. For these three oxides, the threshold grain size for the total suppression of intergranular microfracture during cooling was reported to be $5 \mu \mathrm{m}, 3 \mu \mathrm{m}$, and $1 \mu \mathrm{m}$, respectively (Kuszyk and Bradt, 1973; Cleveland and Bradt, 1978). (iii) In situ observations of intergranular microcracking in notched plates of alumina subjected to cyclic compression at room temperature reveal that the extent of microcracking decreases significantly with decreasing grain size (Suresh and Brockenbrough, 1988). Here microcracking damage in the vicinity of the notch tip and the attendant nucleation of a fatigue crack are strongly influenced by both the residual stresses (arising from thermal and elastic anisotropy) and the imposed cyclic compressive stresses (Suresh, 1991).

There is considerable uncertainty in identifying the conditions that control thermal stress-induced microfracture because of the experimental difficulties in observing the microcracks (especially in fine-grained materials) and the wide variations in compositions and processing conditions used by different investigators. There is general agreement on the existence of a threshold grain size for microcracking and on the deleterious role of coarser grains in exacerbating spontaneous intergranular microfracture during cooling (e.g., Rice and Pohanka, 1979). In keeping with these observations, in our calculations we observe a precipitous increase in the number of cracked facets above a grain size of about $20 \mu \mathrm{m}$, and a certain tendency of coarse grained materials to disintegrate beyond a critical temperature drop. This latter effect, however, may be sensitive to details in the modeling of triple points and be promoted by the two-dimensional character of the formulation.

Although the present analyses pertain to a planar array of equi-axed grains, the trends observed in the statistical properties of the residual stresses can be reasonably expected to carry over to three dimensions. Refinements to the analysis should include the consideration of local stress concentrations at multiple grain junctions, the detailed modeling of microcrack growth through those junctions, and the consideration of three-dimensional grain geometries.

\section{Acknowledgments}

The authors wish to thank Dr. Lynn Ewart for her help with the finite element modeling. This work has been supported by the Materials Research Group on Micro-Mechanics of FailureResistant Materials at Brown University which is funded by the National Science Foundation under grant DMR-9002994. S. S. acknowledges the support of the U.S. Dept. of Energy under grant DE-FG02-84ER-45167.

\section{References}

Blendell, J. E., and Coble, R. L., 1982, "Measurement of Stress Due to Thermal Expansion Anisotropy in $\mathrm{Al}_{2} \mathrm{O}_{3}$," Journal of the American Ceramic Society, Vol. 65, No. 3, pp. 174-78.

Boas, W., and Honeycombe, R. W. K., 1946, "The Plastic Deformation of Non-Cubic Metals by Heating and Cooling," Proceedings of the Royal Society, London, Vol. A186, No. 1, pp. 57-71.

Clarke, F. J. P., 1964, "Residual Strain and the Fracture Stress-Grain Size Relationship in Brittle Solids," Acta Metallurgica, Vol. 12, No. 2, pp. 139-43.

Cleveland, J. J., and Bradt, R. C., 1978, "Grain Size/Microcracking Relation for Pseudo-brookite Oxides," Journal of the American Ceramic Society, Vol. 61, No. $11-12$, pp. 478-81.

Davidge, R. W., and Green, T. J., 1968, "Strength of Two-Phase Ceramic Glass Materials,"' Journal of Materials Science, Vol. 3, No. 6, pp. 629-34.

Evans, A. G., 1978, "Microfracture from Thermal Expansion Anisotropy-

I. Single Phase Systems," Acta Metallurgica, Vol. 26, No, 12, pp. 1845-1853

Fredrich, J. T., and Wong, T.-F., 1986, "Micromechanics of Thermally In duced Cracking in Three Crustal Rocks," Journal of Geophysical Research B. Vol. 91, No. 12, pp. 12,743-12,764.

Fu, Y., and Evans, A. G., 1985, "Some Effects of Microcracks on the Me chanical Properties of Brittle Solids-I. Stress, Strain Relations," Acta Metal. lurgica, Vol. 33, No. 8, pp. 1515-1523.

Kingery, W. D., Bowen, H. K., and Uhlmann, D. R., 1976, Introduction to Ceramics, 2nd ed., John Wiley and Sons, New York.

Kuszyk, J. A., and Bradt, R. C., 1973, "Influence of Grain Size on Effects of Thermal Expansion Anisotropy in $\mathrm{MgTi}_{2} \mathrm{O}_{5}$," Journal of the American $\mathrm{Ce}_{2}$ ramic Society, Vol. 56, No. 8, pp. 420-23.

Laws, N., and Lee, J. C., 1989, "Microcracking in Polycrystalline Ceramics Elastic Isotropy and Thermal Anisotropy," Journal of the Mechanics and Physics of Solids, Vol. 37, No. 5, pp. 603-618.

Matsuo, Y., and Sasaki, H., 1966, "Effect of Grain Size on Microcracking in Lead Titanate Ceramics," Journal of the American Ceramic Society, Vol 49, No. 4, pp. 229-30.

Mood, A. M., Graybill, F. A., and Boes, D. C., 1974, Introduction to the Theory of Statistics, 3rd ed., McGraw-Hill, New York.

Ortiz, M., and Molinari, A., 1988, "Microstructural Residual Stresses in Ceramic Materials," Journal of the Mechanics and Physics of Solids, Vol. 36, No. 4 , pp. $385-400$.

Rice, R. W., and Pohanka, R. C., 1979, "Grain Size Dependence of Spon taneous Cracking in Ceramics," Journal of the American Ceramic Society, Vol. 62, No. 11-12, pp. 559-563.

Simmons, G., and Wang, H., 1971, Single Crystal Elastic Constants and Calculated Aggregate Properties: A Handbook, 2nd ed., MIT Press, Cambridge. MA.

Suresh, S., 1991, Fatigue of Materials, Cambridge University Press, Canbridge, U.K.

Suresh, S., anid Brockenbrough, J. R., 1988, "Theory and Experiments of Fracture in Cyclic Compression: Single Phase Ceramics, Transforming Ceramics and Ceramic Composites," Acta Metallurgica, Vol. 36, No. 6, pp. 1455-1470

Tvergaard, V., and Hutchinson, J. W., 1988, "Microcracking in Ceramic Induced by Thermal Expansion or Elastic Anisotropy," Journal of the A merican Ceramic Society, Vol. 71, No. 3, pp. 157-166. 